\title{
Clinical Study of Cardiovascular Involvement in Patients with Connective Tissue Diseases
}

\author{
Dolly Mathew*, Cicy Bastian \\ Cardiology Medical College, Calicut, India \\ Email: *drdollymathew@gmail.com
}

How to cite this paper: Mathew, D. and Bastian, C. (2017) Clinical Study of Cardiovascular Involvement in Patients with Connective Tissue Diseases. Open Journal of Rheumatology and Autoimmune Diseases, 7, 178-185.

https://doi.org/10.4236/ojra.2017.74018

Received: September 12, 2017

Accepted: November 7, 2017

Published: November 10, 2017

Copyright $\odot 2017$ by authors and Scientific Research Publishing Inc. This work is licensed under the Creative Commons Attribution International License (CC BY 4.0).

http://creativecommons.org/licenses/by/4.0/

\begin{abstract}
Objective: The aim of this study was to evaluate cardiovascular involvement in patients with connective tissue diseases. Methods: A total of 99 patients (mean age $36.6 \pm 10.7$ years) with connective tissue diseases were included in the study. Patients were assessed based on their sex, age, disease duration, cardiovascular symptoms, erythrocyte sedimentation rate, antinuclear antibody, and anti-double stranded DNA antibodies. Moreover, cardiac investigations including electrocardiography (ECG), chest X-ray and echocardiography were performed. Results: The study included 90 (90.9\%) females and 9 (9.09\%) males, aged between 18 and 64 years. Chest pain, palpitation, dyspnoea on exertion, syncope, fatigue, and cardiac murmur were the most cardiac symptoms and signs of patients. ECG abnormalities associated with sinus bradycardia, sinus tachycardia, ST interval and T waves, were found in $27 \%$ patients. Chest X-ray with cardiomegaly was found in $10(10.1 \%)$ patients. Echocardiography abnormalities were observed in $32 \%$ of patients with pericardial involvement, mitral regurgitation, and diastolic dysfunction. Moreover, the incidence of pericardial effusion was found to be significantly $(p<0.05)$ higher in patients with connective tissue diseases. Conclusion: The study indicates the correlation between disease activity, disease duration and abnormal echocardiography of cardiovascular involvement in connective tissue diseases.
\end{abstract}

\section{Keywords}

Cardiovascular Abnormalities, Electrocardiography, Echocardiography

\section{Introduction}

Connective tissue diseases (CTD) are immune-mediated inflammatory diseases involving the cardiovascular system. Inflammatory mediators are associated with many autoimmune diseases including rheumatoid arthritis (RA), systemic lupus 
erythematosus (SLE), ankylosing spondylitis (AS), Behcet's disease, Sjogren syndrome (Sjogr), polymyositis, mixed connective tissue disease (MCTD) and Systemic sclerosis (SSc) [1] [2]. Chronic inflammation can affect blood mononuclear cells, vascular cell adhesion molecule 1, proinflammatory cytokines, and matrix metalloproteinases. Chronic inflammations affected in patients with CTD and lead to development of atherosclerosis [3]. Cardiovascular abnormalities in CTD patients included heart failure, valvular heart disease, coronary artery disease, and pericardial disease [4] [5] [6]. Pericarditis is one of the most common manifestations of cardiovascular involvement in CTD. Furthermore, Valvular disorders, coronary vasculitis, systolic, and diastolic dysfunction can be observed in CTD [7]. In particular, cardiac manifestations were severe and life threatening, often leading to death. The excess of cardiovascular mortality is due to chronic inflammation, duration of autoimmune disease, effects of immunosuppressive therapy, and immunological abnormalities [8]. Antinuclear antibodies (ANA) and anti-double stranded DNA antibodies (anti-dsDNA) antibodies are considered useful in the diagnosis and assessment of the disease activity in RA and SLE patients [9]. Non-invasive techniques which include electrocardiogram (ECG), chest X-ray and echocardiography are the particular processes in detecting cardiovascular abnormalities [10] [11]. ECG is commonly the primary examination for diagnosing cardiovascular disease. A chest X-ray is widely used in the diagnosis of cardiovascular radiographic examination. The presence of different signs was evaluated using chest $\mathrm{X}$-ray including upper zone flow redistribution, lung interstitial edema, alveolar pulmonary edema, bilateral pleural effusion, and cardiomegaly [11]. However, echocardiography has a higher sensitivity for detection of cardiovascular disease [12]. Therefore, the present study was to investigate the extent of cardiac abnormalities associated with CTD patients using clinical examination and non-invasive methods. ECG, chest X-ray, and echocardiography were performed to recognize the degree of cardiovascular involvement in CTD.

\section{Patients and Methods}

\subsection{Study Design}

The study was undertaken at cardiology medical college, Calicut, Kerala, from June 2006-June 2009. This cross-sectional study was conducted in 99 patients presented with connective tissue diseases. The study population fulfilled the American College of Rheumatology (ACR) criteria for CTD [13] [14] [15]. The study protocol was approved by institutional ethical committee (IEC). Written informed consent was procured from every patient. Inclusion of patients in the CTD was based on the following criteria: age $\geq 18$ years, all patients of either sex and clinical diagnosis of CTD verified by a rheumatologist. Exclusion criteria were patients with a history of any congenital heart disease. Detailed history, clinical parameters and cardiovascular symptoms were assessed; Laboratory investigations included a complete haemogram, urine analysis, serum electrolytes, 
fasting blood sugar, lipid profile, renal function test, liver function test, immunological assays as ANA) and anti-dsDNA. ECG, chest X-ray, and echocardiography were also performed. ECG tests were evaluated for each patient and interpreted by a cardiologist. Furthermore, chest X-ray with posteroanterior view of each patient was interpreted by a radiologist. Two-dimension and Doppler (pulsed-wave, continuous-wave, and color flow) echocardiography were applied in standard forms. Pericardial effusion, concentric left ventricular hypertrophy (LVH), valve thickening, regurgitant lesion, mitral regurgitation (MR), aortic regurgitation (AR), Libman sacks vegetation, right ventricular endomyocardial fibrosis (RV EMF) were measured by echocardiography. Echocardiographic dimensions were performed according to guidelines of the American Society of Echocardiography [16].

\subsection{Statistical Analysis}

Statistical analysis was carried out using Microsoft excel spreadsheet (version 2007, Microsoft Corp, Seattle, Washington). Values were expressed as a mean \pm standard deviation or as percentages. The analysis was performed by using chi-square and Fisher's exact test using SPSS software. A p value $<0.05$ was considered statistically significant.

\section{Results}

The age of the patients varied from 18-64 years. The mean age of patients was $36.64 \pm 10.73$ years. Table 1 shows the baseline characteristics of 99 patients manifested with CTD. The disease duration ranged between 1 and 16 years (mean $4.41 \pm 2.89$ years). The mean erythrocyte sedimentation rate in CTD patients was found to be $41.68 \pm 32.76 .13$ (13\%) hypertension patients were diagnosed in the studied population. Out of the 55 RA patients, 46 (83.6\%) patients showed ANA positivity, while $26(81.2 \%)$ patients showed ANA and anti-dsDNA positivity among 32 SLE patients. Chest pain (22\%), palpitation (11\%), dyspnoea on exertion (28\%), syncope (1\%), fatigue (11\%) and cardiac murmur (5\%) were the most common cardiac signs and symptoms of CTD patients as shown in Table 2. In this study, 27 patients had abnormal findings in ECG. As shown in Table 3, 9 (33.3\%) patients with sinus tachycardia and 8 (29.6\%) patients with sinus bradycardia had abnormal findings in ECG. Furthermore, 7 (25\%) patients with ST interval and T wave changes, 2 (7\%) patients with right bundle branch block and in 1 (3\%) patient with complete heart block were found abnormal in ECG. As shown in Table 4, a chest X-ray was abnormal in $12(12.1 \%)$ patients. Cardiomegaly was found in $10(10.1 \%)$ patients. Abnormal Pulmonary artery hypertension was identified in $2(2.02 \%)$ patients. Out of 99 patients, 32 patients were observed abnormal finding in echocardiography. Systemic lupus erythematosis group had a higher pericardial effusion as compare to RA (18.75 vs. $1.8 ; \mathrm{p}=0.006)$. Moreover, $\mathrm{MR}$ and diastolic dysfunction were the most abnormal findings in echocardiography ( $21 \%$ and $25 \%$ patients, 
Table 1. Baseline characteristics of the study population $(n=99)$.

\begin{tabular}{lc}
\hline \multicolumn{1}{c}{ Demographic and baseline characteristics } & $(\mathrm{n}=99)$ \\
\hline Age, Mean \pm SD & $36.64 \pm 10.73$ \\
Duration (years) & $4.41 \pm 2.89$ \\
Gender & $90(90)$ \\
Female & $9(9)$ \\
Male & \\
CTD & $55(55)$ \\
RA & $32(32)$ \\
SLE & $5(5)$ \\
MCTD & $2(2)$ \\
Behcet's & $2(2)$ \\
AS & $1(1)$ \\
Sjogr & $1(1)$ \\
Ploymyositis & $1(1)$ \\
SSc & $41.68 \pm 32.76$ \\
ESR, Mean \pm SD & $13(13)$ \\
HTN & \\
Immune profile, $\mathrm{n}(\%)$ & $46(84)$ \\
ANA positivity; RA (n $=55)$ & $26(81)$ \\
ANA; Anti-dsDNA positivity; SLE $(\mathrm{n}=32)$ & \\
\hline
\end{tabular}

RA-Rheumatoid arthritis, SLE-Systemic lupus erythematosis, MCTD-Mixed connective tissue disease, AS—Ankylosing spondylitis, Sjogr-Sjogren's, SSc-Systemic sclerosis, ESR-Erythrocyte sedimentation rate, HTN-Hypertension, ANA-Antinuclear antibodies, Anti-dsDNA—-anti-double stranded DNA antibodies.

Table 2. Cardiovascular symptom distribution.

\begin{tabular}{ccccc}
\hline Symptom & RA $(\mathrm{n}=55)$ & SLE $(\mathrm{n}=32)$ & MCTD $(\mathrm{n}=5)$ & OTHERS $(\mathrm{n}=7)$ \\
\hline Chest pain & $12(21.8 \%)$ & $7(21.8 \%)$ & $3(60 \%)$ & - \\
Palpitation & $8(14.5 \%)$ & $2(6.2 \%)$ & - & - \\
Dyspnoea on exertion & $17(30.9 \%)$ & $10(31.2 \%)$ & $1(20 \%)$ & - \\
Syncope & $1(1.8 \%)$ & - & - & $1(14.2 \%)$ \\
Fatigue & $4(7.2 \%)$ & $4(12.5 \%)$ & $2(40 \%)$ & - \\
Cardiac murmurs & $4(7.2 \%)$ & $1(3.1 \%)$ & - & - \\
\hline
\end{tabular}

Table 3. ECG findings in the studies patients.

\begin{tabular}{ccccc}
\hline ECG Finding & RA $(\mathrm{n}=55)$ & SLE $(\mathrm{n}=32)$ & Others $(\mathrm{n}=12)$ & p-value \\
\hline Normal & $39(70.9 \%)$ & $21(65.6 \%)$ & $12(1 \%)$ & 0.055 \\
Sinus bradycardia & $5(9.0 \%)$ & $3(9.3 \%)$ & - & 0.9681 \\
Sinus tachycardia & $4(7.2 \%)$ & $5(15.6 \%)$ & - & - \\
RBBB & $2(3.6 \%)$ & - & - & - \\
CHB & $1(1.8 \%)$ & - & - & 0.7263 \\
STT changes & $4(7.2 \%)$ & $3(9.3 \%)$ & - & - \\
\hline
\end{tabular}

ECG-Electrocardiogram, RBBB-Right bundle branch block, CHB—complete heart block. 
Table 4. Chest X-ray findings.

\begin{tabular}{cc}
\hline Chest X-ray & $(\mathrm{n}=99)$ \\
\hline Cardiomegaly & $10(10.1 \%)$ \\
PAH & $2(2.02 \%)$ \\
\hline
\end{tabular}

PAH-Pulmonary artery hypertension.

Table 5. Echocardiographic findings in the studies patients.

\begin{tabular}{ccccc}
\hline Finding & RA $(\mathrm{n}=55)$ & SLE $(\mathrm{n}=32)$ & Others $(\mathrm{n}=12)$ & p-value \\
\hline Pericardial effusion & $1(1.8 \%)$ & $6(18.75 \%)$ & - & 0.0066 \\
Concentric LVH & $1(1.8 \%)$ & - & - & 0.6615 \\
Valve thickening & $1(1.8 \%)$ & $2(6.25 \%)$ & - & 0.4010 \\
Regurgitant lesion & - & - & - & - \\
MR & $2(3.63 \%)$ & $4(12.5 \%)$ & $1(8.33 \%)$ & 0.2934 \\
AR & $1(1.8 \%)$ & - & $1(8.33 \%)$ & 0.2452 \\
Stenotic lesion & - & - & - & - \\
LS vegetation & - & $1(3.12 \%)$ & - & 0.3419 \\
Chamber dialatation & - & $1(3.12 \%)$ & - & 0.3419 \\
Systolic dysfunction & - & $1(3.12 \%)$ & - & 0.3419 \\
Diastolic dysfunction & $5(9.09 \%)$ & $2(6.25 \%)$ & $1(8.33 \%)$ & 0.8942 \\
RV EMF & $1(1.8 \%)$ & - & - & 0.6615 \\
\hline
\end{tabular}

LVH-Left ventricular hypertrophy, MR-Mitral regurgitation, AR-Aortic regurgitation, LS vegetationLibman sacks vegetation, RV EMF-Right ventricular endomyocardial fibrosis.

respectively Table 5). Echocardiographic findings of patients were as follows: 1 (3\%) concentric LVH, 3 (9\%) valve thickening, 2 (6\%) AR, 1 (3\%) Libman-sacks vegetation, 1 (3\%) chamber dilatation, 1 (3\%) systolic dysfunction and 1 (3\%) RV EMF. The mean duration of CTD in patients was 4.41 years. However, echocardiographic abnormalities were detected in 32 patients, 17 (53.12\%) patients with CTD were studied over a period of one to five years, and 15 (46.87\%) patients suffered from CTD more than five years. Moreover, we found a relation between disease duration and echocardiographic abnormalities finding in CTD patients.

\section{Discussion}

In the present study, we investigate clinical correlations and prevalence of cardiovascular dysfunction in patients with CTD. We used ECG, chest X-ray, and echocardiography as a non-invasive technique for evaluation of CTD patients. In patients with CTD, raised ESR is an indirect indication of inflammation and presence of disease activity. This indicates that most CTD patients were in the active stage. Anti-nuclear antibodies and anti-double stranded DNA antibodies are most significant tests in diagnosing RA and SLE. In a recent study, abnormalities in immune regulation were observed in $81 \%$ in SLE and $84 \%$ of RA pa- 
tients. Patients with RA and SLE have been shown elevated antibody levels correlating with disease activity [17]. In Desouky et al. study, that is nearly associate with our study results of antibody level [9]. In this study, we used ECG technique for evaluation of CTD patients. The high frequency of sinus bradycardia, sinus tachycardia, right bundle branch block, ST interval and $\mathrm{T}$ waves changes were seen in RA and SLE patients. Steroid therapy was probably responsible for producing the slowly ascending modify ST-T changes and previously reported increase of ST-T changes in RA and SLE [18] [19] [20]. In a recent study, we found 12 patients with abnormal chest X-ray with cardiomegaly and pulmonary artery hypertension. Our result of chest X-ray abnormality is nearly consistent with Ali et al. study [21]. Echocardiography has played a central role in analyze the morphological and functional change of the cardiac patients with CTD. Cardiovascular involvement was found in 32\% echocardiography patients, including SLE (53\%), RA (21\%) and other (25\%) patients. SLE group had a higher pericardial effusion as compare to RA in echocardiography $(p=0.006)$. Pericardial effusion was the most frequent abnormality in patients [22]. Previous studies showed abnormalities in pericardium, diastolic dysfunction, mitral valve and aortic involvements in echocardiography [23] [24]. In our study, we also detected similar disorders in echocardiography including 7 (21\%) patients with pericardial effusion, 7 (21\%) patients with mitral regurgitation and $8(25 \%)$ patients with diastolic dysfunction in CTD. It seems that patients caused a more evident pericardial effusion, diastolic dysfunction, and mitral regurgitation which may be possible due to longer duration of disease. In our study, we found a relation between disease duration and alteration of pericardial effusion expressed by echocardiography in CTD patients $(\mathrm{p}<0.05)$. These results in our study are similar to the obtained significant results from Masooleh et al. study [7]. The past studies have shown that worsen cardiovascular effect due to disease modifying antirheumatic drugs, non-steroidal anti-inflammatory drugs or steroids therapy in RA patients [25] [26] [27]. These findings suggested that non-invasive cardiac tests for patients might have a role of cardiovascular involvement with CTD for early diagnosis and treatment. The high prevalence of pericardial effusion, diastolic dysfunction, mitral regurgitation, ST interval and $\mathrm{T}$ wave changes in our study can confirm the cardiovascular involvement in CTD patients. Our study has included only the patients presented with CTD, and there were some limitations. This study was performed with cross-sectional analysis and the small study population. Moreover, large-scale prospective studies will need confirmation these findings and management of patients with CTD.

\section{Conclusion}

It is concluded that CTD patients show pericardial effusion characterized by echocardiography. The relation between disease duration and pericardial effusion expressed by abnormal echocardiography suggest cardiovascular involve- 
ment in CTD. Therefore, early detection of cardiovascular abnormalities by using ECG, chest X-ray, and echocardiography may reduce the morbidity and mortality due to cardiovascular involvement in CTD.

\section{Conflicts of Interest}

The authors declare no conflicts of interest.

\section{References}

[1] Pohl, D. and Benseler, S. (2013) Systemic Inflammatory and Autoimmune Disorders. Handbook of Clinical Neurology, 112, 1243-1252. https://doi.org/10.1016/B978-0-444-52910-7.00047-7

[2] Crawford, M.H. (2013) Current Diagnosis and Treatment Cardiology. 4th Edition, McGraw-Hill Education.

[3] Sitia, S., Atzeni, F., Sarzi-Puttini, P., et al. (2009) Cardiovascular Involvement in Systemic Autoimmune Diseases. Autoimmunity Reviews, 8, 281-286. https://doi.org/10.1016/j.autrev.2008.08.004

[4] Gasparyan, A.Y. (2012) Cardiovascular Risk and Inflammation: Pathophysiological Mechanisms, Drug Design, and Targets. Current Pharmaceutical Design, 18, 1447 1449. https://doi.org/10.2174/138161212799504777

[5] Dimitroulas, T., Giannakoulas, G., Karvounis, H., et al. (2014) Micro- and Macrovascular Treatment Targets in Scleroderma Heart Disease. Current Pharmaceutical Design, 20, 536-544. https://doi.org/10.2174/13816128113199990555

[6] Knockaert, D.C. (2007) Cardiac Involvement in Systemic Inflammatory Diseases. European Heart Journal, 28, 1797-1804. https://doi.org/10.1093/eurheartj/ehm193

[7] Shenavar Masooleh, I., Zayeni, H., Haji-Abbasi, A., et al. (2016) Cardiac Involvement in Rheumatoid Arthritis: A Cross-Sectional Study in Iran. Indian Heart Journal, 68, 332-335.

[8] Ungprasert, P., Wannarong, T., Panichsillapakit, T., et al. (2014) Cardiac Involvement in Mixed Connective Tissue Disease: A Systematic Review. International Journal of Cardiology, 171, 326-330.

[9] El Desouky, S.M., El-Gazzar, I.I., Rashed, L.A., et al. (2015) Correlation between Various Clinical Parameters of Systemic Lupus Erythematosus and Levels of Anti-Histone and Anti-Chromatin Antibodies. The Egyptian Rheumatologist, 37, 97-104.

[10] Manes, M.T., Guzzo, D., Rizzo, M., et al. (2006) Cardiovascular Disease in Early Collagen Diseases. Minerva Cardioangiologica, 54, 151-158.

[11] Fonseca, C., Mota, T., Morais, H., et al. (2004) The Value of the Electrocardiogram and Chest X-Ray for Confirming or Refuting a Suspected Diagnosis of Heart Failure in the Community. European Journal of Heart Failure, 6, 807-812, 821-802.

[12] Kim, M.N., Kim, S.A., Kim, Y.H., et al. (2016) Head to Head Comparison of Stress Echocardiography with Exercise Electrocardiography for the Detection of Coronary Artery Stenosis in Women. Journal of Cardiovascular Ultrasound, 24, 135-143. https://doi.org/10.4250/jcu.2016.24.2.135

[13] Tan, E.M., Cohen, A.S., Fries, J.F., et al. (1982) The 1982 Revised Criteria for the Classification of Systemic Lupus Erythematosus. Arthritis \& Rheumatology, 25, 1271-1277. https://doi.org/10.1002/art.1780251101

[14] Arnett, F.C., Edworthy, S.M., Bloch, D.A., et al. (1988) The American Rheumatism 
Association 1987 Revised Criteria for the Classification of Rheumatoid Arthritis. Arthritis \& Rheumatology, 31, 315-324. https://doi.org/10.1002/art.1780310302

[15] Vitali, C., Bombardieri, S., Moutsopoulos, H.M., et al. (1993) Preliminary Criteria for the Classification of Sjogren's Syndrome. Results of a Prospective Concerted Action Supported by the European Community. Arthritis \& Rheumatology, 36, 340-347. https://doi.org/10.1002/art.1780360309

[16] Sahn, D.J., DeMaria, A., Kisslo, J., et al. (1978) Recommendations Regarding Quantitation in M-Mode Echocardiography: Results of a Survey of Echocardiographic Measurements. Circulation, 58, 1072-1083.

https://doi.org/10.1161/01.CIR.58.6.1072

[17] Simon, J.A., Cabiedes, J., Ortiz, E., et al. (2004) Anti-Nucleosome Antibodies in Patients with Systemic Lupus Erythematosus of Recent Onset. Potential Utility as a Diagnostic Tool and Disease Activity Marker. Rheumatology (Oxford), 43, 220-224. https://doi.org/10.1093/rheumatology/keh024

[18] Stewart, I.M. and Marks, J.S. (1977) E.C.G. Abnormalities in Steroid-Treated Rheumatoid Patients. The Lancet, 2, 1237-1238.

[19] Laitinen, O., Kentala, E. and Leirisalo, M. (1978) Electrocardiographic Findings in Patients with Connective Tissue Disease. Scandinavian Journal of Rheumatology, 7, 193-198. https://doi.org/10.3109/03009747809095653

[20] Geraldino-Pardilla, L., Gartshteyn, Y., Piña, P., et al. (2016) ECG Non-Specific ST-T and QTc Abnormalities in Patients with Systemic Lupus Erythematosus Compared with Rheumatoid Arthritis. Lupus Science \& Medicine, 3.

https://doi.org/10.1136/lupus-2016-000168

[21] Biharas Monfared, A., Agha Farajollah, S., Sabour, F., et al. (2015) Comparison of Radiological Findings of Chest X-Ray With Echocardiography in Determination of the Heart Size. Iranian Red Crescent Medical Journal, 17, e18242. https://doi.org/10.5812/ircmj.18242

[22] Sagristà-Sauleda, J., Mercé, A.S. and Soler-Soler, J. (2011) Diagnosis and Management of Pericardial Effusion. World Journal of Cardiology, 3, 135-143.

https://doi.org/10.4330/wjc.v3.i5.135

[23] CoŞKun, S., ÖZoran, K., Mermerci, B., et al. (2005) Cardiac Involvement in Patients with Rheumatoid Arthritis. APLAR Journal of Rheumatology, 8, 23-31. https://doi.org/10.1111/j.1479-8077.2005.00118.x

[24] Di Franco, M., Paradiso, M., Mammarella, A., et al. (2000) Diastolic Function Abnormalities in Rheumatoid Arthritis. Evaluation by Echo Doppler Transmitral Flow and Pulmonary Venous Flow: Relation with Duration of Disease. Annals of the Rheumatic Diseases, 59, 227-229. https://doi.org/10.1136/ard.59.3.227

[25] Firestein, G.S. (2017) Chapter 69 Etiology and Pathogenesis of Rheumatoid Arthritis, in Kelley and Firestein's Textbook of Rheumatology (Tenth Edition). Elsevier, e1117, 1115-1166.

[26] Hajas, A., Szodoray, P., Nakken, B., et al. (2013) Clinical Course, Prognosis, and Causes of Death in Mixed Connective Tissue Disease. The Journal of Rheumatology, 40, 1134-1142. https://doi.org/10.3899/jrheum.121272

[27] Pieringer, H. and Pichler, M. (2011) Cardiovascular Morbidity and Mortality in Patients with Rheumatoid Arthritis: Vascular Alterations and Possible Clinical Implications. QJM, 104, 13-26. https://doi.org/10.1093/qjmed/hcq203 